\title{
Peripheral absolute threshold spectral sensitivity in retinitis pigmentosa
}

\author{
ROBERT W. MASSOF, MARY A. JOHNSON, AND \\ DANIEL FINKELSTEIN \\ From the Wilmer Ophthalmological Institute, Johns Hopkins University School of Medicine, \\ Baltimore, USA
}

SUMMARY Dark-adapted spectral sensitivities were measured in the peripheral retinas of 38 patients diagnosed as having typical retinitis pigmentosa (RP) and in 3 normal volunteers. The patients included those having autosomal dominant and autosomal recessive inheritance patterns. Results were analysed by comparisons with the CIE standard scotopic spectral visibility function and with Judd's modification of the photopic spectral visibility function, with consideration of contributions from changes in spectral transmission of preretinal media. The data show 3 general patterns. One group of patients had absolute threshold spectral sensitivities that were fit by Judd's photopic visibility curve. Absolute threshold spectral sensitivities for a second group of patients were fit by a normal scotopic spectral visibility curve. The third group of patients had absolute threshold spectral sensitivities that were fit by a combination of scotopic and photopic spectral visibility curves. The autosomal dominant and autosomal recessive modes of inheritance were represented in each group of patients. These data indicate that RP patients have normal rod and/or cone spectral sensitivities, and support the subclassification of patients described previously by Massof and Finkelstein.

Perimetric absolute threshold measures, employing a short wavelength and a long wavelength stimulus, yielded data suggesting there may be subgroups of dominant and recessive retinitis pigmentosa (RP), primarily differing in the relation of rod sensitivity to cone sensitivity throughout the visual field. ${ }^{1}$ One group of patients, class 1 , behaved as if only cones mediated detection at absolute threshold over the entire visual field; there was no evidence of rod function. A second group of patients, class 2, behaved as if rods mediated detection at absolute threshold in the extrafoveal visual field, despite threshold elevations as great as $4 \log$ units; these results suggest concomitant loss of rod and cone sensitivity. A third group of patients, class 3, behaved as if rod sensitivity was reduced relative to cone sensitivity, thereby allowing cones to make greater contributions to detection at absolute threshold. The interpretation of the 2-colour threshold data and the subsequent identification of the RP subgroups depend on the assumption that RP patients have normal rod and/or cone spectral sensitivities.

Correspondence to Dr R. Massof, Wilmer, B-34, Johns Hopkins Hospital, Baltimore, MD 21205, USA.
Berson et al. ${ }^{2}$ measured dark-adapted spectral sensitivities at $7^{\circ}$ superior field in $4 \mathrm{RP}$ patients from one autosomal dominant pedigree and in 3 confirmed RP patients from a second pedigree, described as autosomal dominant with reduced penetrance. ${ }^{3}$ For the first group of patients spectral sensitivity curves were fitted well by the Commission Internationale de l'Eclairage (CIE) scotopic spectral visibility function. For the second group of patients spectral sensitivity curves approximated Wald's photopic spectral visibility function. ${ }^{4}$

Zeavin and Wald $^{5}$ reported spectral sensitivity measures from one autosomal dominant RP patient recorded at $5^{\circ}$ and $10^{\circ}$ inferior field. The $5^{\circ}$ data were similar to the normal scotopic spectral sensitivity curve and the $10^{\circ}$ data resembled a normal photopic spectral sensitivity curve.

Employing reflection densitometry Ripps et al. ${ }^{6}$ found rhodopsin-shaped density difference spectra at $45^{\circ}$ temporal retina for 3 RP patients from a single autosomal dominant pedigree. Also using reflection densitometry Alpern (personal communication) has recorded a rhodopsin-like bleach action spectrum from one RP patient at $18^{\circ}$ temporal retina.

The results of the above-cited studies support the notion that RP patients have normal rod and 


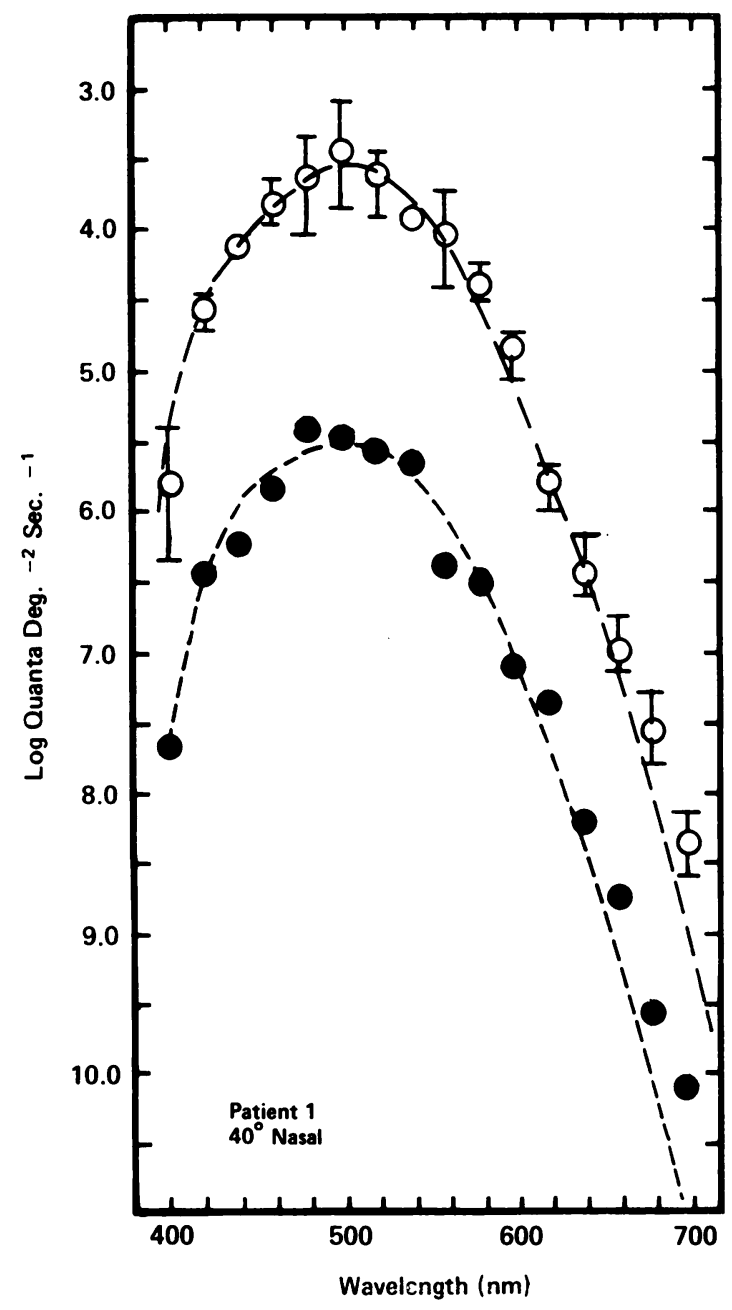

Fig. 1 Dark-adapted spectral sensitivities of normal observers and RP patient 1 obtained at $40^{\circ}$ in the nasal field. For all figures the open circles represent the means of 3 normal observers, the error bars encompass the range of normal sensitivities at each wavelength, and the closed circles represent data obtained from the RP patients. All normal data are fitted by the CIE scotopic visibility function. In this figure data from RP patient 1 are fitted by the CIE scotopic visibility function.

cone spectral sensitivities. However, no data from recessive pedigrees have been reported, and there are some limitations and anomalies to be considered. Ripps et $a .^{6}{ }^{6}$ observed a shift toward longer wavelengths of the density difference spectrum at $30^{\circ}$ for 2 patients. This shift possibly reflects cone contributions to the density measures, but the argument also could be made that it reflects an abnormality

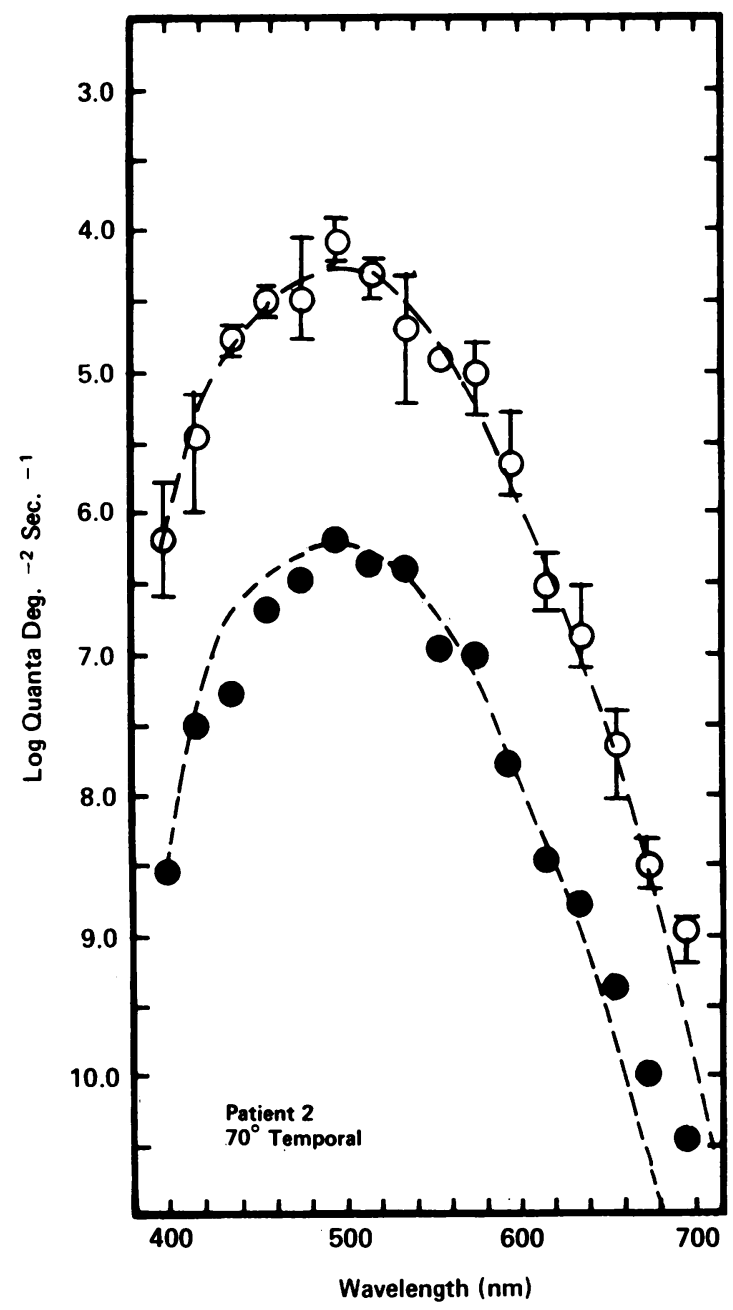

Fig. 2 Symbols have the same meaning as in Fig. 1. Spectral sensitivities were obtained at $70^{\circ}$ in the temporal field. Data from patient 2 are fitted by the CIE scotopic visibility function adjusted for an increase in lens yellowing by a factor of $1 \cdot 5$.

in the absorption spectrum of the rod visual pigment. The spectral sensitivity curves measured by Berson et $a l^{2}{ }^{2}$ on the first dominant pedigree were made with broad-band colour filters, leaving the spectral resolution less than desirable. An interference wedge was employed for the measures on the second group of patients, ${ }^{3}$ but the interpretation of the data is difficult because the fit to Wald's photopic visibility curve is only approximate; there are significant departures for 2 of the 3 patients. $^{3}$ Zeavin and Wald ${ }^{5}$ did not describe how their spectral sensitivity measures were made. Though the 'rod' 
and 'cone' functions for their patient appear to have sensitivity peaks in the expected spectral regions, no comparison with normal spectral sensitivity curves were made.

The purpose of this paper is to report our measures of dark-adapted threshold spectral sensitivity in the peripheral retina of patients representing the previously described putative subtypes of dominant and recessive RP. ${ }^{1}$ The results are analysed by comparisons with the Commission Internationale de l'Eclairage (CIE) standard scotopic spectral visibility function, ${ }^{7}$ Judd's modification $^{8}$ of the CIE photopic spectral visibility function, and considerations of contributions from changes in preretinal media spectral transmission.

\section{Materials and methods}

\section{SUBJECTS}

Absolute threshold spectral sensitivity curves were determined for 38 patients diagnosed as having typical $\mathbf{R P}^{\mathbf{1}}$ and 3 normal volunteers. Data are reported for 14 representative cases described in Table 1. Informed consent was obtained from each participant in the study.

\section{A P PARATUS}

One channel of a multichannel Maxwellian view apparatus was employed for the spectral sensitivity measures. A 1000 watt xenon arc lamp served as the source. The arc was imaged on the entrance slit of a Spex double-grating monochromator (blazed at $500 \mathrm{~nm}$ ) with a spectral slit width of $2.5 \mathrm{~nm}$ and a

Table 1 Characteristics of patients with retinitis pigmentosa

\begin{tabular}{cllllll}
\hline Patient & Age & Sex & $\begin{array}{l}\text { Mode of } \\
\text { inheritance }\end{array}$ & VA & Lens & Group \\
\hline 1 & 38 & F & Dominant & $20 / 20$ & Clear & 2 \\
2 & 35 & M & Simplex & $20 / 30$ & Subtle PSC & 2 \\
3 & 14 & F & Simplex & $20 / 20$ & Clear & 2 \\
4 & 22 & F & Simplex & $20 / 25$ & $1+$ PSC & 2 \\
5 & 38 & M & Simplex & $20 / 40$ & Clear & 2 \\
6 & 13 & F & Dominant & $20 / 30$ & Clear & 1 \\
7 & 29 & F & Recessive & $20 / 25$ & $1+$ PSC & 1 \\
8 & 22 & M & Simplex & $20 / 30$ & $1+$ PSC & 1 \\
9 & 56 & F & Dominant & $10 / 150$ & $2+$ PSC & 1 \\
10 & 36 & F & Recessive & $20 / 40$ & $1+$ PSC & 1 \\
11 & 39 & F & Recessive & $20 / 25$ & Subtle PSC & 3 \\
12 & 11 & M & Dominant & $20 / 30$ & Clear & 3 \\
13 & 23 & F & Dominant & $20 / 50$ & Clear & 3 \\
14 & 12 & F & Dominant & $20 / 30$ & Clear & 3
\end{tabular}

"Group identifies absolute threshold pattern as described in the text. VA = visual acuity. $\mathbf{P S C}=$ posterior subcapsular cataract. scattered light level of $10^{-9}$. A balanced inconel (alloy) neutral density wedge and auxiliary neutral density filters, both calibrated at each wavelength, served to control the intensity of the monochromatic beam. The monochromator exit slit was focused on a $1 \mathrm{~mm}$ diameter limiting aperture and chopped at $1 \mathrm{~Hz}$ by a silent shutter. The limiting aperture image was collimated and passed through a 4 dioptre final lens, thereby producing a retinal image subtending a visual angle of $2 \cdot 3^{\circ}$.

The observer was positioned on a dental impression bite-bar such that the limiting aperture was imaged in the centre of the dilated ocular entrance

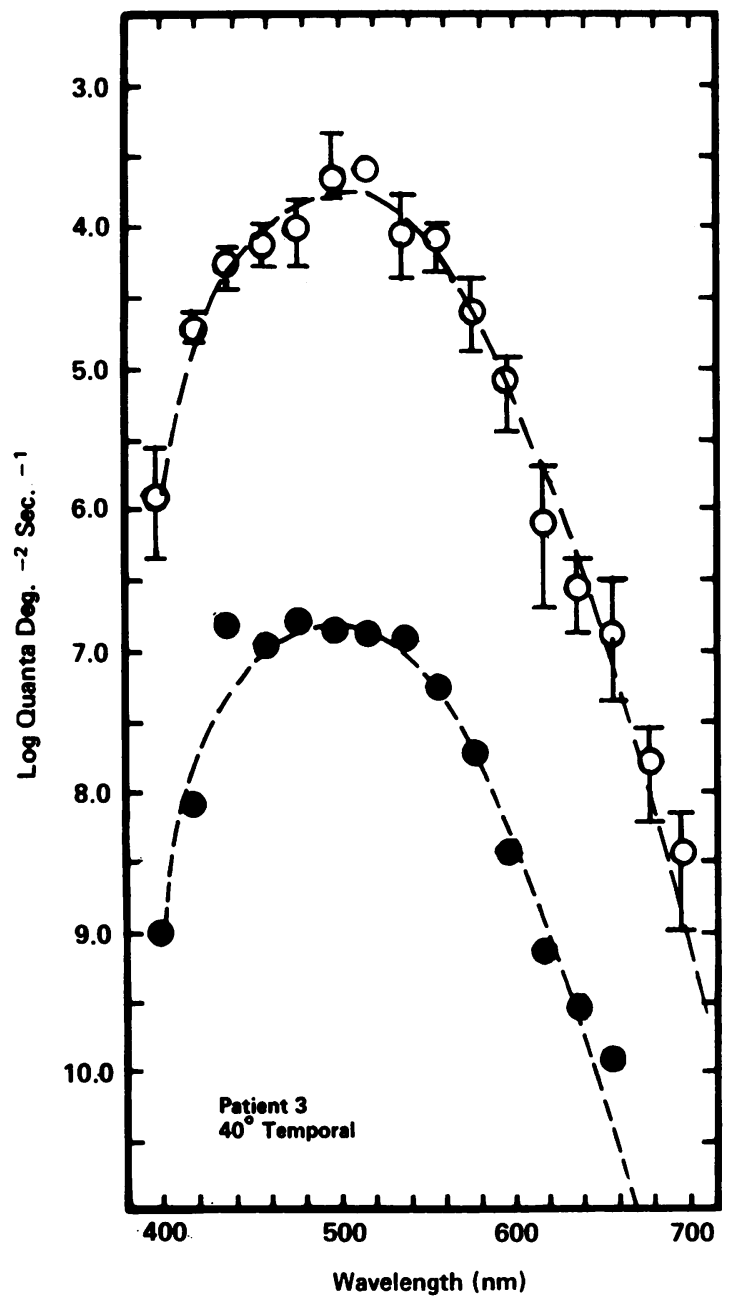

Fig. 3 Symbols have the same meaning as in Fig. 1. Spectral sensitivities were obtained at $40^{\circ}$ in the temporal field. Data from RP patient 3 are fitted by the CIE scotopic visibility function, adjusted for an increase in lens yellowing by a factor of 1.25. 
pupil. The $\mathrm{x}, \mathrm{y}, \mathrm{z}$ bite-bar positioning apparatus was mounted on a rotary indexing table with the centre of rotation located in the limiting aperture focal plane. Therefore, when the observer was properly aligned, the observer's entrance pupil was at the centre of rotation of the indexing table. A red fixation cross was provided, and the observer was rotated until the field stop was imaged at the desired retinal eccentricity.

\section{PROCEDURE}

The subject adapted to the dark for at least 45 minutes prior to the data collection session. Absolute

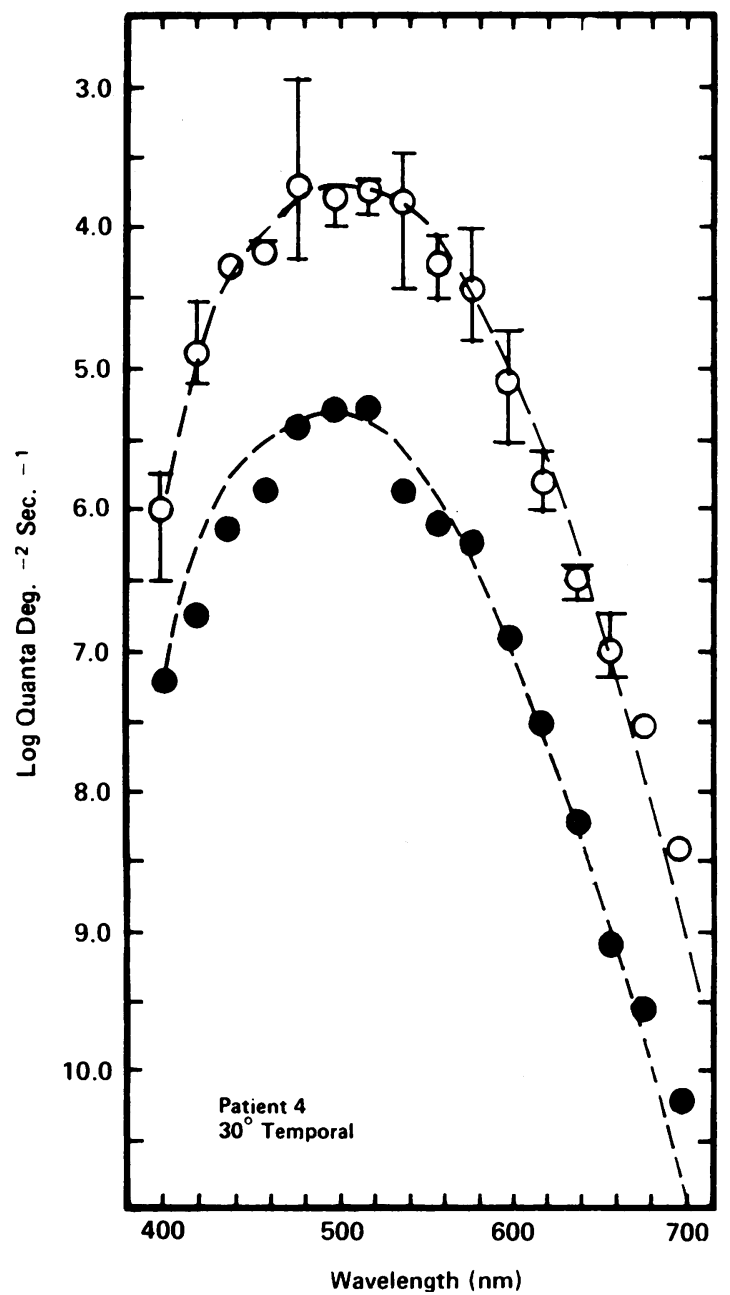

Fig. 4 Symbols have the same meaning as in Fig. 1. Spectral sensitivities were obtained at $30^{\circ}$ in the temporal field. Data from RP patient 4 are fitted by the CIE scotopic visibility function. thresholds then were measured at each of 16 wavelengths from $400 \mathrm{~nm}$ to $700 \mathrm{~nm}$ in $20 \mathrm{~nm}$ steps. The wavelengths were presented in a pseudorandom order, and threshold was determined by slowly and continuously increasing the intensity of the stimulus until the subject reported detecting the 'flashing light'. This procedure was repeated 3 to 5 times (depending on response variability) before the wavelength was changed.

The data collection session lasted between 30 and 60 minutes, depending on the pace of the observer. It was not possible to obtain more than one session of data on the RP patients. Conse-

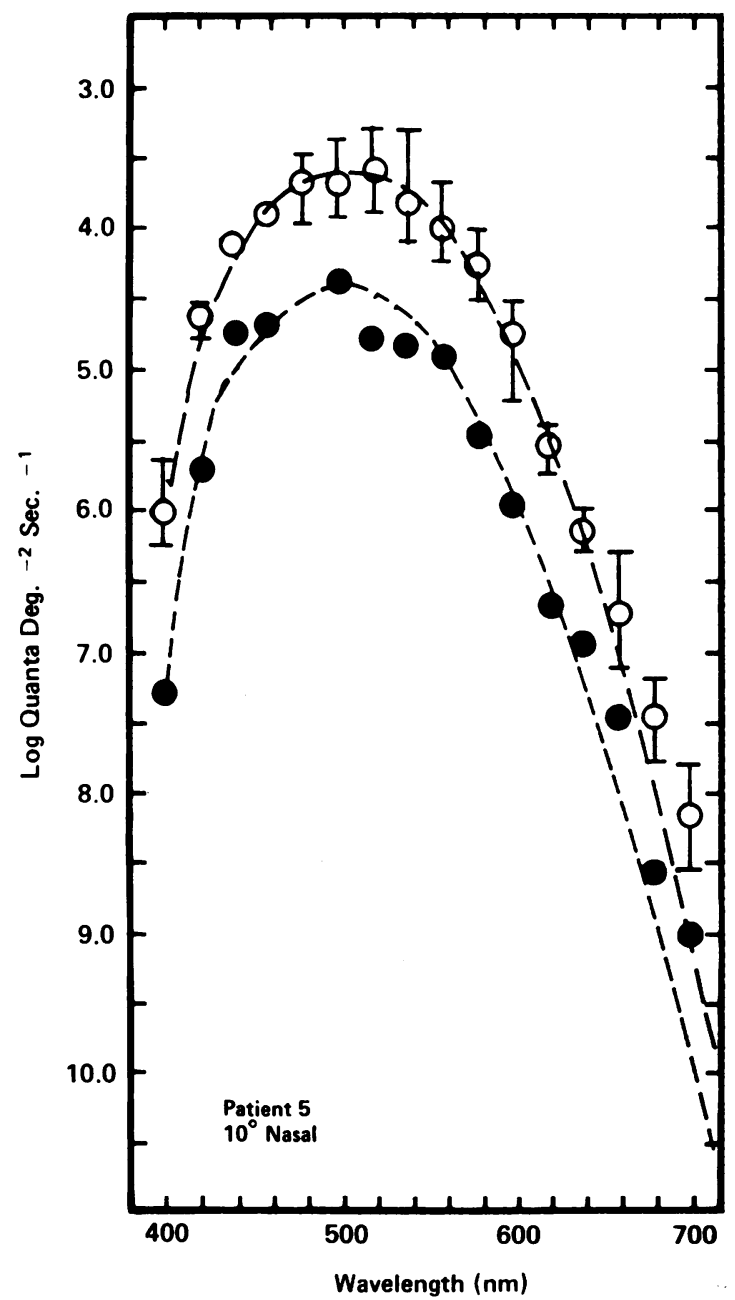

Fig. 5 Symbols have the same meaning as in Fig. 1. Spectral sensitivities were obtained at $10^{\circ}$ in the nasal field. Data from RP patient 5 are fitted by the CIE scotopic visibility function, adjusted for an increase in lens yellowing by a factor of 1.75 . 
quently no estimates of between-session variability are available.

\section{Results}

NORMAL OBSERVERS

Absolute threshold spectral sensitivities were measured at the following field positions: $10^{\circ}, 30^{\circ}, 40^{\circ}$, and $70^{\circ}$ temporal and $10^{\circ}, 15^{\circ}, 30^{\circ}$, and $40^{\circ}$ nasal, for each of the 3 normal observers. These eccentricities were chosen to correspond with those sampled in the group of patients. The same proce-

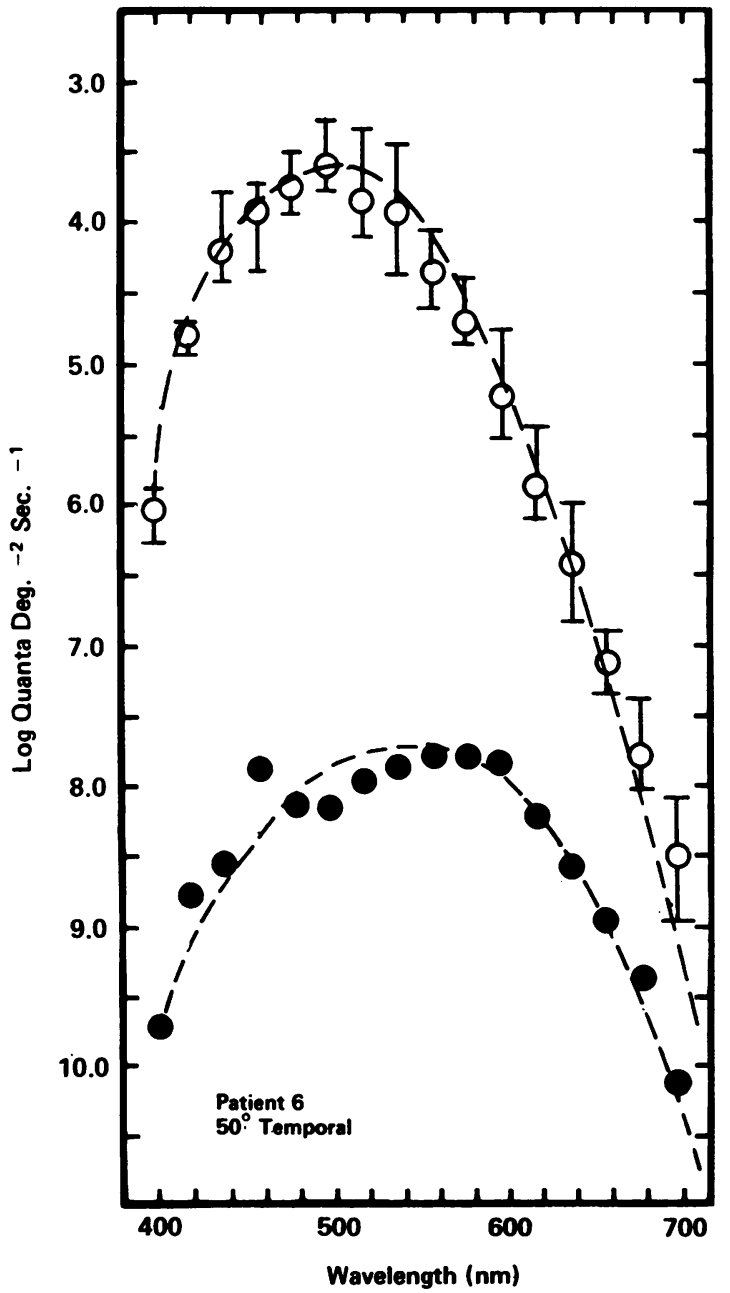

Fig. 6 Symbols have the same meaning as in Fig. 1. Spectral sensitivities were obtained at $50^{\circ}$ in the temporal field. Data from RP patient 6 are fitted by $J$ Judd's modification of the CIE photopic spectral visibility function, adjusted for the absence of macular pigment at $50^{\circ}$. dures were followed as those described for the RP patients, and the data for each field position were collected in a single session.

Figs. 1 to 14 illustrate the means and ranges of the data for the 3 normal observers (open circles). In each figure the continuous curve plotted along with the normal data represents the CIE scotopic spectral visibility function. ${ }^{\text {? }}$

RP PATIENTS

There are 3 obvious factors that could serve to alter dark-adapted spectral sensitivity in RP: (1)

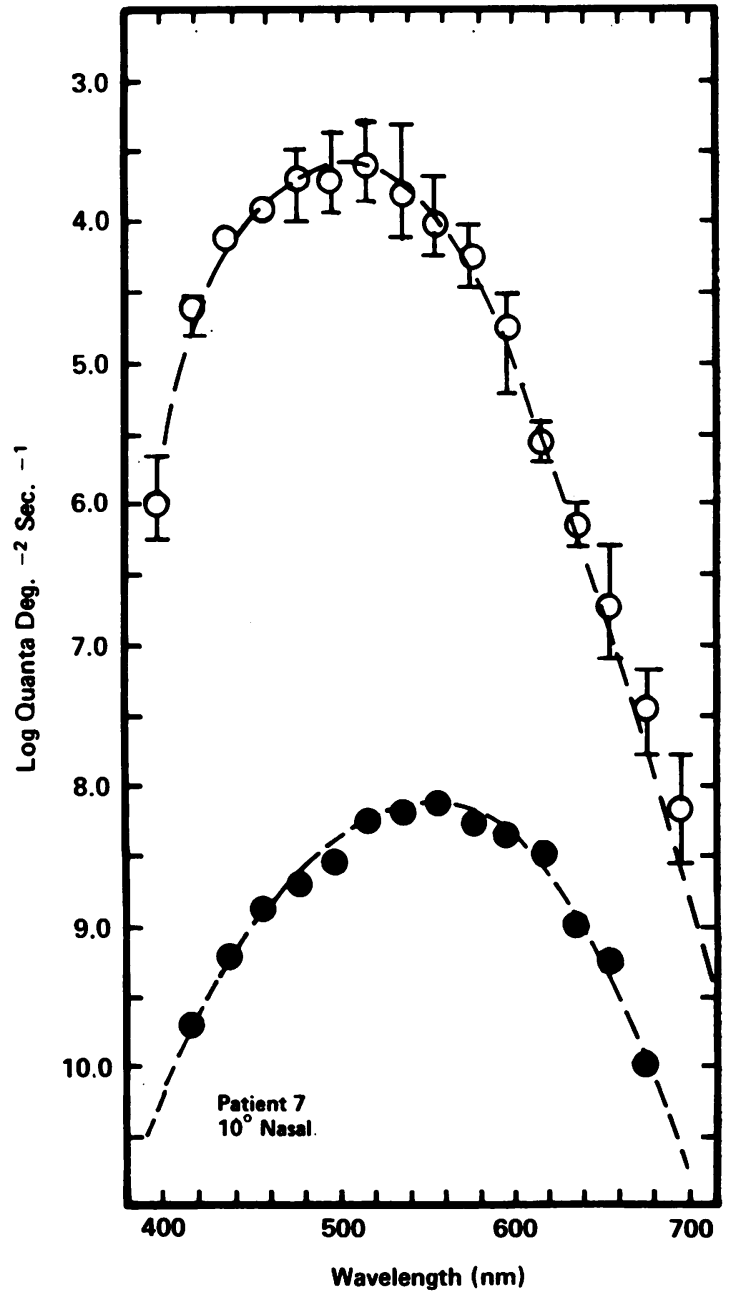

Fig. 7 Symbols have the same meaning as in Fig. 1. Spectral sensitivities were obtained at $10^{\circ}$ in the nasal field. Data from RP patient 7 are fitted by Judd's modification of the CIE photopic spectral visibility function, adjusted for a $50 \%$ decrease in concentration of macular pigment at $10^{\circ}$. 
an anomaly in the spectral absorption of the rod visual pigment, (2) a change in the spectral transmission of the preretinal ocular media, and (3) a preferential reduction in rod sensitivity, thereby allowing cones to increase their contribution to threshold measures. We will consider each of these factors in analysing the RP spectral sensitivity data.

Results for 1 dominant and 4 simplex (or recessive) RP patients, who show class 2 threshold behaviour (implying concomitant losses of rod and cone sensitivity ${ }^{1}$ ) are illustrated in Figs. 1 to 5, along with the normal data for the specified retinal

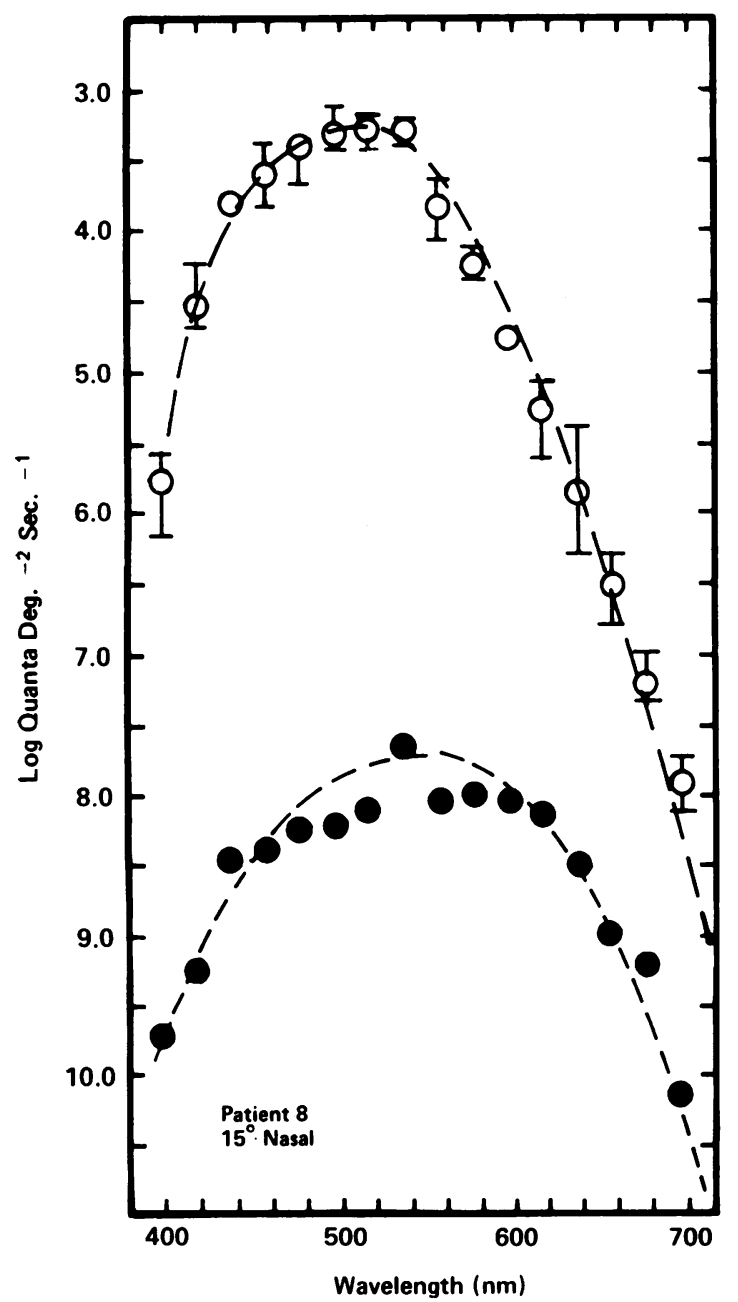

Fig. 8 Symbols have the same meaning as in Fig. 1. Spectral sensitivities were obtained at $15^{\circ}$ in the nasal field. Data from RP patient 8 are fitted by Judd's modification of the CIE photopic spectral visibility function, adjusted for the absence of macular pigment in this patient at $15^{\circ}$. position. The continuous curve through the RP data represents the CIE standard scotopic visibility curve. The excellent fit of the standard curve to the RP patient data suggests normal rhodopsin spectral absorption underlying these RP spectral sensitivities, despite the 1 to $3 \log$ unit threshold elevations. Abnormally large cone contributions to threshold would be expected to produce a departure from the scotopic function or an inflection in the long wavelengths.

To fit the data of patients 2,3 , and 5 it was necessary to correct the standard curves for in-

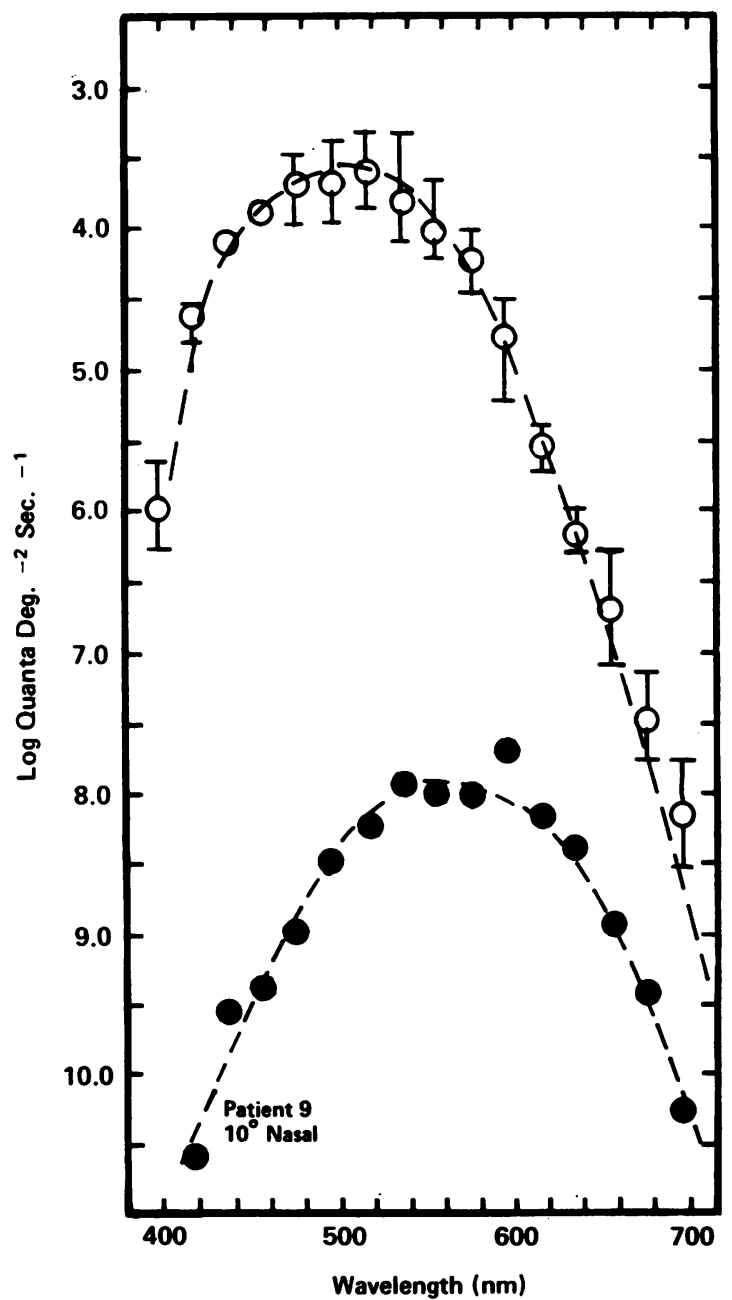

Fig. 9 Symbols have the same meaning as in Fig. 1. Spectral sensitivities were obtained at $10^{\circ}$ in the nasal field. Data from $R P$ patient 9 are fitted by Judd's modification of the CIE photopic visibility function, adjusted for an increase in lens yellowing by a factor of 4 and for a $60 \%$ decrease in concentration of macular pigment at $10^{\circ}$. 
creased lens optical density. The largest lens density change in this group of patients was for patient 5 , who required an increase by a factor of 1.75 .

Figs. 6 to 10 illustrate results for 2 dominant and 3 recessive or simplex RP patients who show class $1^{1}$ threshold behaviour (implying no rod contributions to threshold measures), plotted along with the appropriate normal data. The continuous curve fitted to the RP data in Figs. 6 to 9 is Judd's photopic spectral visibility function, ${ }^{8}$ corrected for changes

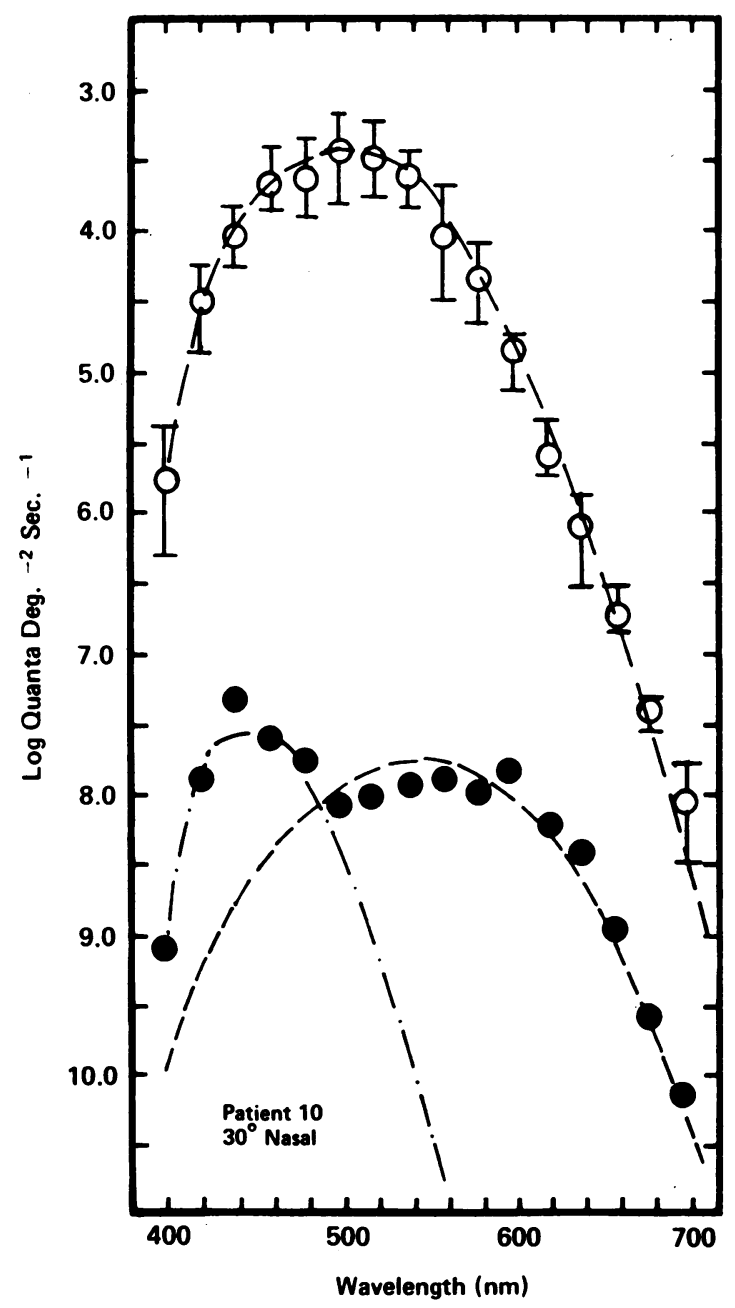

Fig. 10 Symbols have the same meaning as in Fig. 1. Spectral sensitivities were obtained at $30^{\circ}$ in the nasal field. Data from RP patient 10 are fitted in the long wavelengths by Judd's modification of the CIE photopic spectral visibility function and in the short wavelengths by the Smith and Pokorny B primary, both adjusted for the lack of macular pigment at $30^{\circ}$. in macular pigment density with retinal position.? To fit the data of patient 9 (Fig. 9) it was necessary to correct Judd's photopic curve for a 4-fold increase in lens density. This patient had a moderately dense $(2+)$ posterior subcapsular cataract.

For patient 10 (Fig. 10) the photopic visibility function fits the long wavelength data (solid curve), but there is a second sensitivity peak in the short wavelengths. These data are surprisingly similar to Weale's ${ }^{9}$ normal peripheral spectral sensitivity

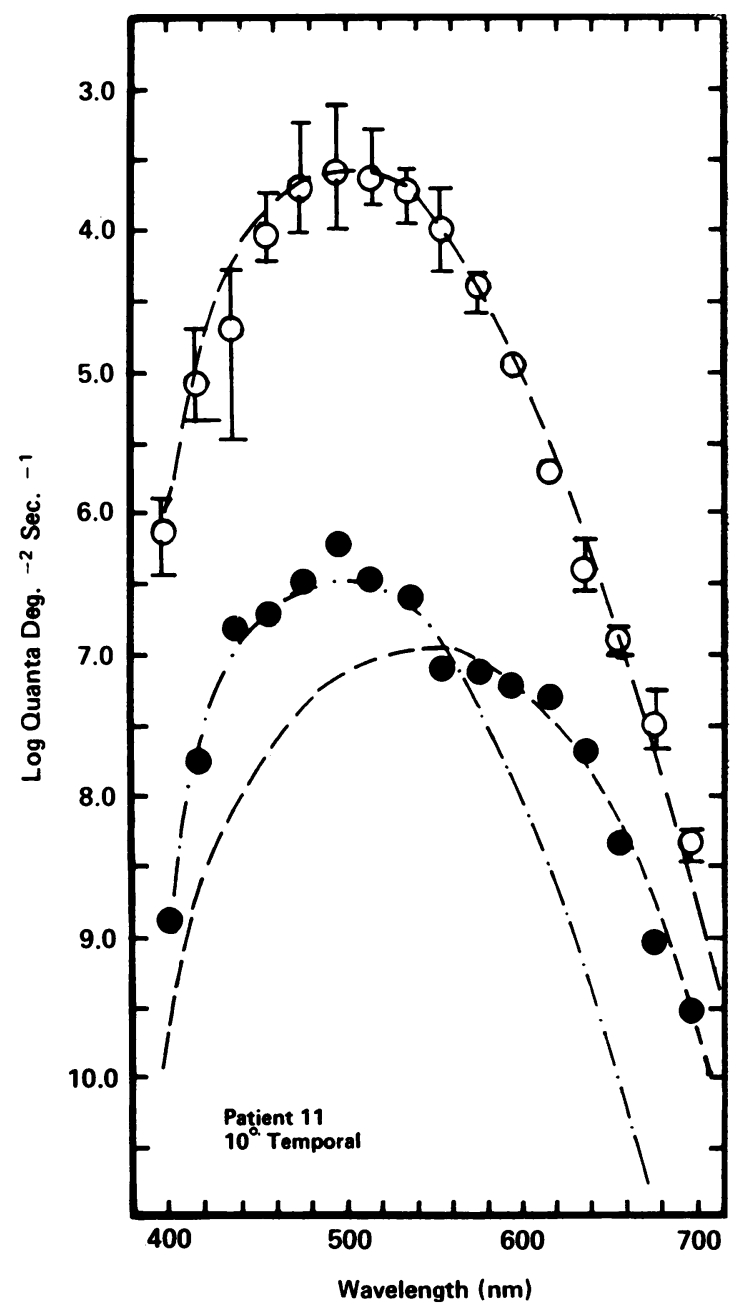

Fig. 11 Symbols have the same meaning as in Fig. 1. Spectral sensitivities were obtained at $10^{\circ}$ in the temporal field. Data from RP patient 11 are fitted in the long wavelengths by Judd's modification of the CIE photopic spectral visibility function adjusted for lack of macular pigment in this patient at $10^{\circ}$, and in the short wavelengths by the CIE scotopic spectral visibility function. Both functions were adjusted for an increase in lens yellowing by a factor of 1.5 . 
functions measured on a white background. By adding the Smith and Pokorny ${ }^{10} \mathrm{~B}$ primary, corrected for the lack of macular pigment at $30^{\circ}$ (dotted curve), we obtain a satisfactory description of the data.

Figs. 11 to 14 illustrate results for 3 dominant and 1 recessive RP patient who showed class $3^{1}$ threshold behaviour (combination of rod and cone contributions to detection). In all these cases the data are well fitted by a combination of the scotopic and

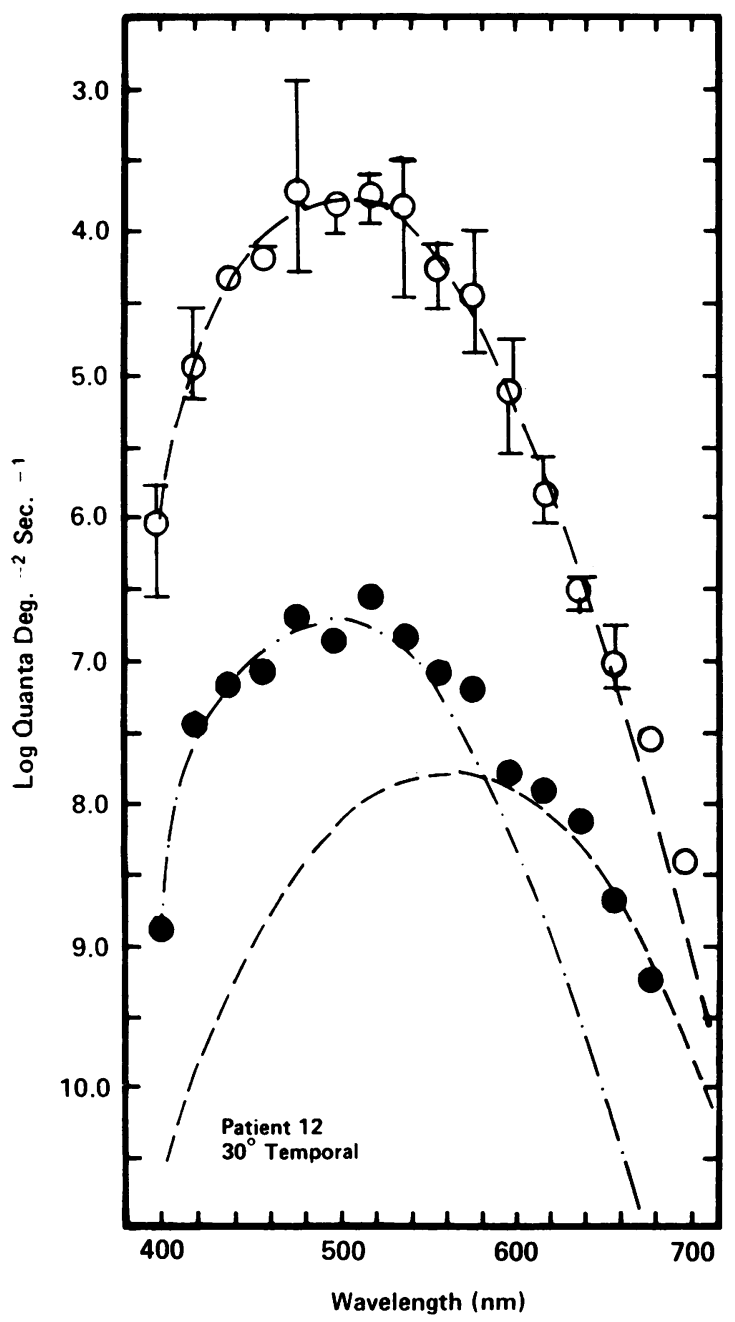

Fig. 12 Symbols have the same meaning as in Fig. 1. Spectral sensitivities were obtained at $30^{\circ}$ in the temporal field. Data from RP patient 12 are fitted in the long wavelengths by Judd's modification of the CIE photopic spectral visibility function adjusted for lack of macular pigment at $30^{\circ}$, and in the short wavelengths by the CIE scotopic spectral visibility function. photopic spectral visibility functions. Lens density corrections were made to fit the data of patients 11 and 14.

\section{Discussion}

Our results indicate that the RP patients who show class 2 threshold behaviour have normal scotopic spectral sensitivity, modified in some cases by greater yellowing of the lens than for the CIE standard

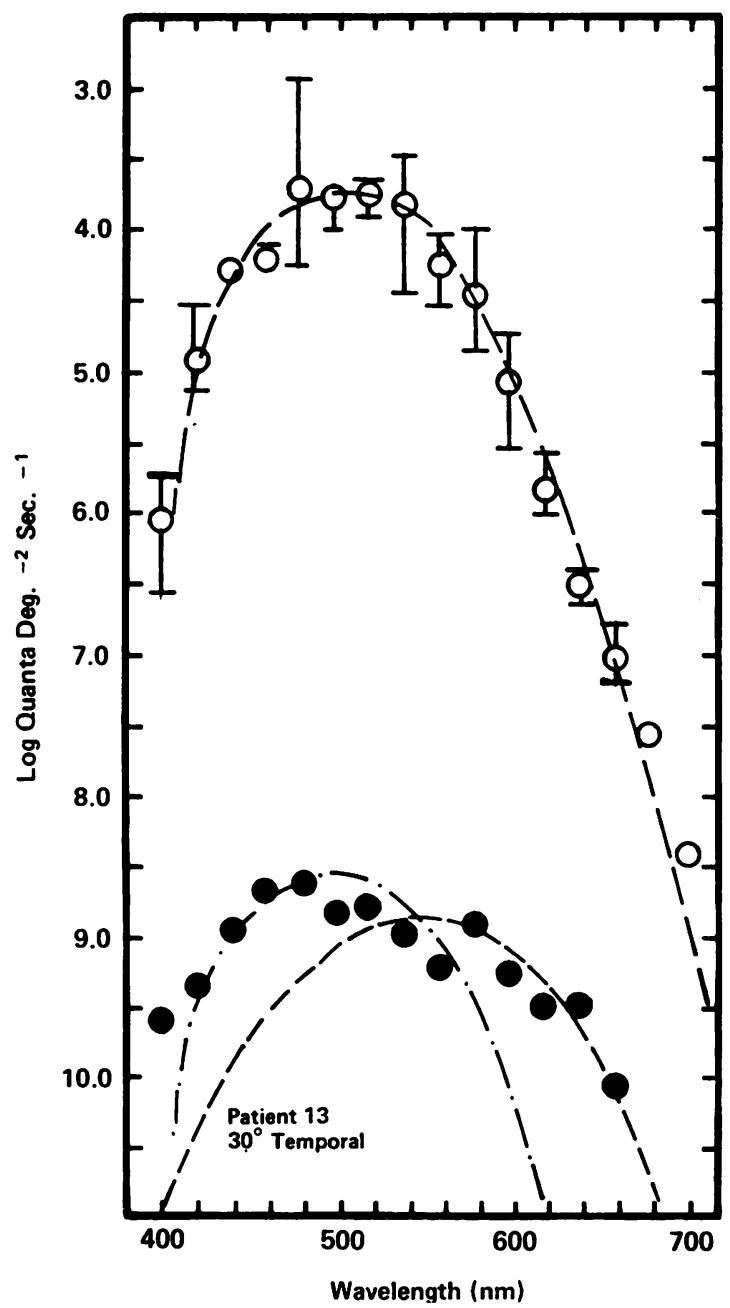

Fig. 13 Symbols have the same meaning as in Fig. 1. Spectral sensitivities were obtained at $30^{\circ}$ in the temporal field. Data from RP patient 13 are fitted in the long wavelengths by Judd's modification of the CIE photopic spectral visibility function adjusted for lack of macular pigment at $30^{\circ}$, and in the short wavelengths by the CIE scotopic visibility function. 
observer. As argued before, measures of scotopic spectral sensitivity in the presence of absolute threshold elevations imply a concomitant loss of rod and cone sensitivity, that is, the relation of rod sensitivity to cone sensitivity remains normal.

The determination of normality of the cone spectral sensitivity functions of the RP patients who showed class 1 threshold patterns is difficult owing to the still existing controversy over the shape of

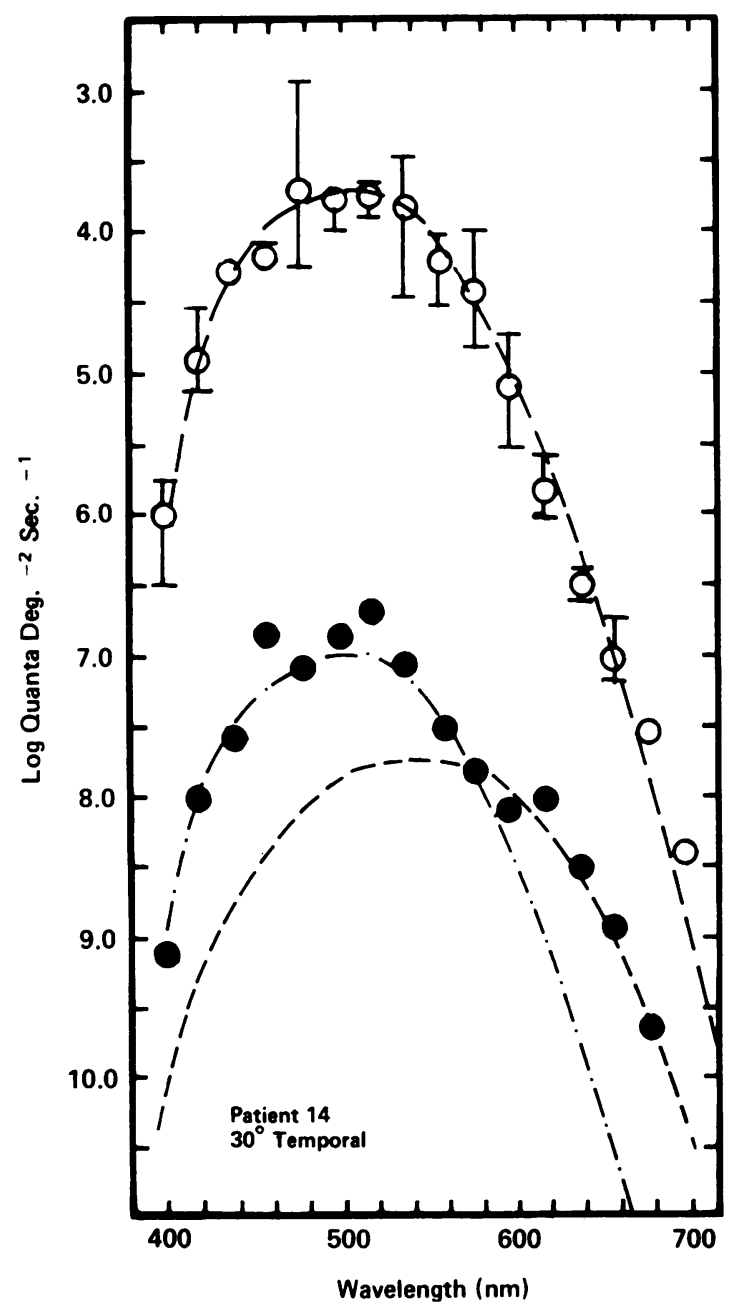

Fig. 14 Symbols have the same meaning as in Fig. 1. Spectral sensitivities were obtained at $30^{\circ}$ in the temporal field. Data from RP patient 14 are fitted in the long wavelengths by Judd's modification of the CIE photopic visibility function adjusted for lack of macular pigment at $30^{\circ}$, and in the short wavelengths by the CIE scotopic visibility function. Both functions were adjusted for an increase in lens yellowing by $a$ factor of $1 \cdot 25$. the normal photopic spectral visibility curve for the peripheral retina. ${ }^{1112}$ Wooten and Wald ${ }^{13}$ demonstrated that apparently the same cone visual pigments are distributed throughout the retina, but relative sensitivites and interactions may change with eccentricity. Weale ${ }^{9}$ found a short-wavelength sensitivity peak at increasing eccentricities, but Wooten et al..$^{11}$ argued that this was due to chromatic adaptation from his background source and that the dark-adapted peripheral spectral sensitivity function is nearly identical to the foveal function. Most of the data we obtained on class 1 RP patients (e.g., Figs. 6-9) agree with this conclusion; however, we obtained spectral visibility curves from 3 RP patients (Fig. 10) that showed a prominent shortwavelength peak for the dark-adapted eye. Keeping in mind that our cases represent a retinal pathology, we consider the similarity to Weale's data may suggest the possibility of normal individual differences. Nevertheless, with the exception of Abramov and Gordon's ${ }^{12}$ data that are similar to the scotopic visibility curve ${ }^{14}$ all peripheral photopic spectral visibility functions agree in the shape of the long wavelength cone pigment. ${ }^{10}$ Therefore, we might argue that peripheral cone spectral senstivities are normal in this group of RP patients and that there is no evidence of rod contributions to threshold at any wavelength.

The results for the final group of patients, who showed class 3 threshold patterns, are accounted for by assuming that the normal scotopic spectral visibility function determines short-wavelength thresholds and the normal photopic spectral visibility function determines long-wavelength thresholds. This accounting is identical to the conclusion drawn earlier about threshold behaviour for these patients. It is not possible to say from these data that scotopic functions are normal in the long wavelengths and photopic functions are normal in the short wavelengths, or that the data do not represent a bizarre new pigment that coincidentally looks like a combination of photopic and scotopic functions. However, adaptation experiments on these patients, employing the 2-colour threshold procedure (to be reported elsewhere), suggest that the latter concern is unfounded.

We have concluded that normal scotopic and photopic spectral visibility functions can be used to describe the spectral sensitivity data of the various types of RP patients. However, before concluding that the spectral sensitivity functions measured here reflect normal visual pigments we must acknowledge the limitations of our data: wavelength steps were $20 \mathrm{~nm}$, only one session of data was obtainable, and the observers were untrained. If there were small shifts in the pigment absorption spectra, for example, 
of about $10 \mathrm{~nm}$, we probably would not be able to resolve them. Furthermore, there are small departures in the long wavelengths from the scotopic visibility function for patients 1-5 (Figs. 1-5). Though these may reflect cone intrusions, we cannot at present say they are significantly greater than those seen for the normal data. They are, however, clearly not of the same order as the cone intrusions seen for patients 11-14 (Figs. 11-14). Despite these caveats, we conclude that the data of this study support our initial premise in the evaluation of 2-colour absolute threshold measures.

This work was supported by research grants from the US Public Health Service, National Institutes of Health (EY01791), and the National Retinitis Pigmentosa Foundation.

\section{References}

1 Massof RW, Finkelstein D. Rod sensitivity relative to cone sensitivity in retinitis pigmentosa. Invest Ophthalmol Visual Sci 1979; 18: 263-72.

2 Berson EL, Gouras P, Gunkel RD. Rod responses in retinitis pigmentosa, dominantly inherited. Arch Ophthalmol 1968; 80: 58-67.

3 Berson EL, Gouras P, Gunkel RD, Myrianthopoulos
NC. Dominant retinitis pigmentosa with reduced penetrance. Arch Ophthalmol 1969; 81 : 226-34.

4 Wald G. Human vision and the spectrum. Science 1945; 101: 653-8.

5 Zeavin BH, Wald G. Rod and cone vision in retinitis pigmentosa. Am J Ophthalmol 1956; 42: 253-69.

6 Ripps H, Brin KP, Weale RA. Rhodopsin and visual threshold in retinitis pigmentosa. Invest Ophthalmol Visual Sci 1978; 17: 735-45.

7 Wyszecki G, Stiles WS. Color Science. New York: Wiley, 1967.

8 Judd DB. Colorimetry and artificial daylight. Proc 12th Session CIE, Stockholm 1, Tech. Commun., No. 7, 1951: 11 .

9 Weale RA. Spectral sensitivity and wavelength discrimination of the peripheral retina. J Physiol 1953; 119: 170-90.

10 Smith VC, Pokorny J. Spectral sensitivity of the foveal cone photopigments between 400 and $500 \mathrm{~nm}$. Vision Res 1975; 15: 161-71.

11 Wooten BR, Fuld K, Spillmann L. Photopic spectral sensitivity of the peripheral retina. J Opt Soc Am 1975; 65: $334-42$.

12 Abramov I, Gordon J. Color vision in the peripheral retina. I. Spectral sensitivity. J Opt Soc Am 1977; 67: 195-202.

13 Wooten BR, Wald G. Color-vision mechanisms in the peripheral retinas of normal and dichromatic observers. J Gen Physiol 1973; 61 : 125-45.

14 Stabell B, Stabell V. Extrafoveal spectral sensitivity during dark adaptation. J Opt Soc Am 1980; 70: 81-6. 